\title{
Philosophiques
}

\section{La grammaticalité des textes littéraires}

\section{Arthur Clayborough}

Volume 8, numéro 1, avril 1981

URI : https://id.erudit.org/iderudit/203151ar

DOI : https://doi.org/10.7202/203151ar

Aller au sommaire du numéro

Éditeur(s)

Société de philosophie du Québec

ISSN

0316-2923 (imprimé)

1492-1391 (numérique)

Découvrir la revue

Citer cet article

Clayborough, A. (1981). La grammaticalité des textes littéraires. Philosophiques, 8(1), 93-111. https://doi.org/10.7202/203151ar d'utilisation que vous pouvez consulter en ligne.

https://apropos.erudit.org/fr/usagers/politique-dutilisation/ 


\title{
LA GRAMMATICALITÉ DES TEXTES LITTÉRAIRES ${ }^{1}$
}

\author{
par Arthur Clayborough
}

Que l'on me permette tout d'abord de demander en quoi un texte littéraire est littéraire. L'affirmation que tout texte peut être considéré comme de la littérature ne surprend pas de nos jours. On implique par là que ce qui compte, c'est le point de vue du lecteur, et si on accepte cette affirmation, abandonnant donc l'idée qu'il y a quelque chose de spécial dans les textes littéraires en tant que tels, la question la plus intéressante est alors de savoir si on doit cesser, en toute bonne logique, de considérer un texte sous un autre angle, quand on le considère comme littéraire. Est-il possible, par exemple, de lire un traité sur les baleines et de l'apprécier, en tant que tel et en tant qu'oeuvre littéraire, dans une seule et même lecture? Prendre plaisir à la lecture d'une oeuvre littéraire, qu'est-ce que cela veut dire? Quand on éprouve du plaisir à lire Victor Hugo ou Charles Dickens, est-ce qu'on apprécie leurs écrits en tant que littérature? Il est assez facile de distinguer entre le plaisir que l'on trouve à lire un ouvrage comme simple lecteur et celui qu'on y trouve en tant que critique. Est-ce que le fait d'apprécier une oeuvre en tant que littérature est réservé à ce second type d'approche? Est-ce qu'elle implique un examen plus explicite des effets esthétiques de l'oeuvre? Signet-on un contrat, si j'ose m'exprimer ainsi, qui nous obligerait à tout regarder sous l'angle de l'esthétique - comme on le fait en entrant au Louvre - quand on se plonge dans une oeuvre littéraire? Allons plus loin encore: doit-on parler de plusieurs types de littérature, ou n'y a-t-il qu'une «littérature»? Lire De l'origine des espèces de Darwin «en tant que littérature», est-ce la même chose que de lire Flaubert ou Wells?

1. Avec référence spéciale à Dickens, Bleak House, chapitre 11. Ce texte a éré traduit de l'anglais par Odile Halmöy. 
Après tout, il semble bien qu'il y ait une nette différence entre le plaisir esthétique que procure, disons, un tas de paquets de flocons de mais ou une roue de bicyclette dans un musée d'art moderne, et celui que procure un Rubens ou un Renoir. Ce second type d'objet a été conçu délibérément pour être apprécié esthétiquement, de sorte que l'artiste et le spectateur peuvent communiquer: l'oeuvre comporte certains éléments qui favorisent cette optique spéciale. Roman Jakobson parle du caractère opaque de l'oeuvre littéraire: elle est enjolivée, individualisée, et c'est le langage particulier de la poésie, par exemple, qui met en évidence sa nature esthétique. Supposons que l'on vous présente un objet en bois de forme étrange, provenant de fouilles archéologiques, et que l'on vous demande quelle était sa fonction. Cet objet comportera peut-être des éléments d'ornementation, qui seront là pour l'embellir et procurer un plaisir esthétique, mais cela ne signifiera pas nécessairement que l'objet lui-même n'ait eu qu'une fonction esthétique. Ces éléments d'ornementation pourront nous permettre, accessoirement et par extrapolation, de replacer l'objet en question dans une tradition, un contexte qui nous fourniront des indications précieuses. Si cet objet n'est pas décoré de la sorte, on s'intéressera à sa forme, à ses «traits structuraux», et, tout en cherchant des détails qui nous mettent sur la voie, on regardera s'il est «bien travaillé», poli à la perfection, etc., et, dans une optique plus pratique, on essaiera de déterminer s'il a la forme d'un manche d'épée ou d'un chandelier. Cette première approche n'est pas très éloignée de ce qu'on appelle une "perspective autonome», où l'oeuvre est examinée en termes de sa "structure interne», avec le moins de référence possible à une intention ou à un objectif avoués.

L'avantage qu'il y a à prendre un exemple simple comme celui que je viens d'utiliser, c'est que, dans l'analyse finale, il permet de voir que ces différentes techniques sont complémentaires. C'est dans un contexte plus étroit seulement - quand on connaît bien l'oeuvre à analyser, les conditions de sa création aussi bien que les intentions de son auteur - que l'on peut s'offrir le luxe de se passer de ces diverses techniques, et considérer l'oeuvre exclusivement sous tel ou tel angle. D'où la querelle entre l'éccle socio-pragmatique, qui considère l'intention sous-tendant les actes de parole, et l'école structuraliste, qui considère la littérature comme un objet autonome. 
Je voudrais essayer de résumer brièvement les difficultés auxquelles on s'est généralement heurté en cherchant à identifier ce qui est «littéraire» ou ce qu'est la littérature.

1. Pour certains, c'est une question de perspective: tout dépend de la «lecture» que l'on fait de l'oeuvre. L'approche littéraire d'un texte demande une interprétation, une exégèse, alors qu' une approche linguistique est purement descriptive. Inutile de discuter ce point de vue ici - je ne fais que présenter cette opposition, caractéristique des deux types d'approche. Pour d'autres, l'oeuvre littéraire est différente des autres textes, soit par sa qualité, soit parce qu'elle comporte davantage de traits particuliers que les autres textes, qui peuvent d'ailleurs eux aussi présenter ces traits particuliers, mais en moins grand nombre. Cette conception d'un caractère «immanent» de la littérature me semble graviter autour de trois idées centrales: (a) L'idée d'unité. L'oeuvre littéraire a une unité organique, c'est un tout organisé. Ses diverses parties sont intégrées à un ensemble qu'elles soustendent. (b) L'idée d'unicité. L'oeuvre littéraire n'est pas seulement un échantillon, représentant un groupe, ce n'est pas non plus un texte dont le seul objectif est de transmettre un message. L'oeuvre littéraire a une valeur propre, elle est comme un vitrail - c'est la métaphore standard -, faite non pour qu'on regarde à travers elle, mais pour qu'on la regarde, elle. C'est là, au fond, ce que suggère Roman Jakobson. (c) L'idée de généralisation. L'oeuvre littéraire va «au-delà des mots»: elle a une portée générale, universelle, en ce qu'elle est souvent une parabole. On a le sentiment qu'elle implique plus que ce qu'elle ne dit. Pour prendre un exemple, si un article de journal rapportant un accident de la circulation peut inciter le lecteur à la prudence quand il se retrouvera au volant, c'est un effet secondaire, en quelque sorte. Le rapport en soi n'est que descriptif. Une oeuvre littéraire traitant le même sujet suggérera plus nettement qu'il s'agit d'un message personnel, d'une de te fabula. Une fois encore, j'essaie simplement d'esquisser à grands traits les principaux courants de pensée, sans chercher à les défendre.

Si l'on accepte l'idée que l'objet de notre enquête - spécifier en quoi l'oeuvre littéraire est littéraire - est limité par l'approche «subjective» ou «pragmatique» d'une part, et par l'approche «autonome» ou «immanente» de l'autre, il reste à considérer les 
intersections éventuelles entre ces deux types d'approche. On peut soutenir: (1) qu'elles s'excluent mutuellement; (2) qu'elles sont nécessairement complémentaires; (3) que ce sont des approches générales, entre lesquelles on peut choisir selon les cas, l'approche sélectionnée comme approche générale faisant éventuellement appel à l'autre en cas de besoin; et enfin, bien sûr, (4) que ni l'un ni l'autre de ces deux types d'approche n'est acceptable.

C'est au troisième de ces points de vue que va ma préférence, et je choisis l'approche pragmatique comme première approche. Cela permet, me semble-t-il, de dire que tout texte - ou presque, ne soyons pas trop catégorique - peut être envisagé sous l'angle de l'esthétique, et aussi que les textes littéraires dans un sens plus étroit présentent en général un certain nombre de traits caractéristiques facilement reconnaissables. On peut de la sorte faire le départ entre, disons, Shakespeare et Charles Darwin, ou, (mutatis mutandis), entre un magnifique ustensile de cuisine et une sculpture de Rodin.

Un autre genre intéressant, c'est la forme de littérature ou d'art extrêmement sophistiquée qui a pour objectif délibéré de tomber entre deux chaises, comme les amoncellements «esthétiques» de paquets de flocons de mais d'Andy Warhol, ou, disons, le mode d'emploi d'un aspirateur présenté comme oeuvre "littéraire». Une caractéristique de cette catégorie est que, quel que soit l'objet sur lequel se porte l'attention, on le présente délibérément dans un contexte qui suggère qu'il est à regarder comme oeuvre d'art ou oeuvre littéraire. Supposons que vous ayez critiqué le dessin des jambes arrière d'un cheval dans un tableau apparemment naturaliste, et qu'on vous dise: "mais c'est une déformation voulue de la perspective!», vous vous retrouveriez dans un univers à la Warhol ou à la Robbe-Grillet, où le sol se dérobe sous vos pieds. Contrairement à ce qui se passe dans le cas de Darwin, où on est libre de décider, dans une certaine mesure, quels traits peuvent être présentés comme «littéraires» - élégance du style, éclat des images, etc. - et contrairement à ce qui est le cas pour Shakespeare, où les traits «littéraires» sont fixés par la tradition, ce troisième type d' «oeuvre littéraire» ne laisse pas de place à la subjectivité et ne fournit pas non plus de critères traditionnels d'évaluation. Il vise au paradoxe, et est en soi d'un 
grand intérêt. On peut comparer l'attrait qu'il exerce au phénomène linguistique de l'aberration pragmatique («je savais qu'il était de Paris, mais j'avais tort»). Une telle oeuvre, qui change délibérément l'univers du discours, est parallèle en quelque sorte au paradoxe du Russell. Elle laisse le lecteur sur sa faim, et l'oblige à repenser son attitude vis-à-vis des conventions courantes. Waismann compare l'aberration pragmatique au déplacement d'un pion d'échec au-delà des limites de l'échiquier:

Essayer de penser quelque chose qui n'ait pas de sens, c'est comme essayer de déplacer une pièce du jeu d'échecs au-delà des limites de l'échiquier. Je peux le faire, mais la règle du jeu ne prévoit pas ce qui se passera alors. Et de la même façon, je peux dire le rouge est industrieux, mais mon intention, en prononçant cette phrase, n'est pas claire (Principles, p. 39).

Les limites de l'échiquier linguistique sont beaucoup moins absolues que celles du jeu d'échec. Des phrases et des pièges littéraires de ce genre peuvent nous conduire non seulement au-delà de l'échiquier, mais aussi à travers le miroir, où, on s'en souvient, les pièces du jeu d'échec ont leur logique propre:

«Et vous, quel genre de choses vous rappelez-vous le mieux?» hasarda Alice.

«Oh, les choses qui se sont passées dans deux semaines», répondit la reine d'un ton négligent.

La fascination qu'a exercée Lewis Carroll sur les philosophes de la linguistique évoquerait peut-être les phrases "thérapeutiques» absurdes de Wittgenstein, du type «Est-ce qu'une douleur consciente fait plus de mal dans l'esprit conscient qu'une douleur inconsciente dans l'esprit inconscient?", dont l'intention était, selon l'expression énigmatique de Wittgenstein, de «montrer à la mouche le moyen de sortir de la bouteille" (to show the fly the way out of the fly-bottle) $)^{1}$.

1. Cf., par ex., Pbilosopbical Investigations, p. 222 e: “Un nouveau-né n'a pas de dents.»-- «Une oie n'a pas de dents." - «Une rose n'a pas de dents." Cette dernière phrase est vraie de toute évidence, pourrait-on dire. Elle est même plus vraie que dans le cas de l'oie. - Et pourtant, elle n'est pas très claire. Car où se trouveraient les dents de la rose? L'oie n'en a pas dans sa màchoire. Et ni l'une ni l'autre bien sûr n'en ont dans leurs ailes; mais personne ne pense à cela en disant qu'elles n'ont pas de dents. - Mais supposez que l'on dise: la vache mastique sa nourriture et puis en asperge la rose, de sorte que la rose a des dents dans la mâchoire d'un animal. Cela ne serait pas absurde parce que personne ne sait par avance où chercher les dents chez une rose. 
La thérapie de Wittgenstein était essentiellement une thérapie logique: mais l'utilisation anomale du langage peut certainement comporter un côté émotionnellement thérapeutique. C'est ce à quoi Izaak $\mathrm{O}$. Revzin fait allusion dans son article Generative Grammars, Stylistics and Poetics:

Les membres de l'OPOJAZ et du cercle linguistique de Moscou ont déjà clairement démontré la différence essentielle entre l'automation de l'emploi quotidien du langage, relié aux mécanismes inconscients de la formation de la langue, et la singularité du langage poétique, qui fait appel à une langue consciemment ou inconsciemment transformée, devant libérer le destinataire d'une perception automatisée et lui permettre de sentir la signification de chaque élément du discours pris à part (p. 568).

Même si cela peut paraître un peu chauvin, on comprend l'idée' de Revzin: s'il y a une thérapie logique dans des formes comme au Nord-Est du pôle Nord, il est au moins aussi vraisemblable qu'il y ait une thérapie émotionnelle dans des expressions comme celles, souvent citées, de Dylan Thomas: a grief ago, ou once below a time, ou the man in the wind and the west moon. La phrase de Chomsky, d'incolores idées vertes dorment furieusement, devenue désormais «proverbiale, pour ainsi dire» comme l'observe Quirk, peut facilement se comprendre comme une sorte de variation sur les vers de Marvell:

Annihilating all that's made

To a green thought in a green shade.

Ziff remarque que ces vers ne posent aucun problème au lecteur féru de poésie ${ }^{2}$. Revzin, ayant appris de Roman Jakobson que D.H. Hymes avait écrit un poème avec pour premier vers la phrase de Chomsky, a tenté d'en faire une version russe. Je pense que nous devons voir là quelque part une morale: pour la tirer, j’emprunterais volontiers à Jung son terme de régressif. Pénétrer dans un univers autre donne un sentiment de libération. Tout texte littéraire constitue un tel univers. Les phrases anomales les plus cohérentes, qui sont facilement assimilées dans le contexte plus étendu d'une oeuvre littéraire ou d'un conte populaire (il n'y a pas de raison pour que les corbeilles à papier ne ronflent pas violemment dans des histoires comme celles de Hans Andersen,

2. Le "vert incolore" ne poserait pas de problème non plus aux enfants habitués à la vieille plaisanterie: "sky blue pink with yellow dots in: ciel bleu rose taché de jaune." 
par exemple), suggèrent fréquemment ce que Louisa Gradgrind appelle «un élan ardent vers une région où les lois, les figures et les définitions ne soient pas absolues ${ }^{3}{ }$. Il est indéniable que cet élan vers une telle région est familier aux étudiants de la littérature: c'est, répétons-le, dans les textes littéraires que les déviations lexicales et logiques du langage se produisent le plus fréquemment. Il est difficile de dire ce que le linguiste peut tirer de tout cela, bien que Revzin soutienne (p. 569) que la linguistique ne peut se développer avec succès sans un contact très étroit avec la stylistique et la poétique. Je pourrait résumer mon point de vue ici en disant que le problème de la grammaticalité me semble inextricablement lié aux problèmes sémantiques: on ne peut séparer les problèmes sémantiques de la relation production/reconnaissance (c'est-à-dire de la pragmatique): et cette relation, en fin de compte, $c$ 'est en liaison avec les textes littéraires qu'on peut l'étudier dans ses formes les plus subtiles, et dans des matériaux bien plus durables que dans les énoncés éphémères de la langue quotidienne.

2. J'ai fait remarquer qu'on peut dire de toute oeuvre d'art qu'elle constitue un univers de discours séparé. Je crois que c'est dans cette direction qu'il faut chercher une solution au problème langue/parole tel qu'il s'applique à la littérature, à la question de savoir si on peut, à la manière de Whorf, considérer Shakespeare, disons, comme constituant en quelque sorte une «langue».

On ne saurait aborder ici ce que sont les interrelations terminologiques entre une langue et un univers de discours; je me limiterai à ce dernier terme, en l'utilisant, bien sûr, dans un sens assez particulier. Ce que je veux faire valoir surtout, c'est que l'oeuvre littéraire est un acte de langage et qu'il faut permettre à chaque «univers» de ce genre de nous présenter les normes par lesquelles il doit être perçu. Je poursuivrai en distinguant les niveaux auxquels chaque texte demande d'être examiné: mais qu'on me permette à ce moment critique de dire que, jusqu'ici, nous nous sommes surtout intéressé aux formes anomales, et qu'il nous faut maintenant considérer l'envers de la médaille, la création des normes. René Wellek, dans Style in Language, proteste vigoureusement contre la tendance qu'on peut avoir à considérer le style littéraire en termes d'écarts par rapport au langage ordinaire, ce qu'il appelle «une sorte de contre-grammaire»:

3. Cf. Hard Times, livre 2, chap. 12. 
Le danger de la stylistique linguistique est qu'elle centre son intérêt sur les déviations par rapport à la norme linguistique. On obtient une sorte de contre-grammaire, une science des écarts. La stylistique normale est abandonnée au grammairien, et la stylistique déviationnelle, réservée aux étudiants en littérature. Mais ce sont souvent les éléments linguistiques les plus courants, les plus normaux qui sont les constituants de la structure littéraire. Une stylistique littéraire se concentrera sur la fin esthétique de chaque appareil linguistique, sur la façon dont il sert un tout (1968, p. 417-18).

Envisagée ainsi, à la lumière de la totalité qu'elle dessert, chaque déviation peut être considérée comme une partie de la norme. M.A.K. Halliday dit de même qu'«il n'y a pas de différence essentielle entre la déviation par rapport à une norme et la création d'une autre norme ${ }^{4} »$. Comme le dit Lewis Carroll, «plus on s'éloigne de l'Angleterre, plus on se rapproche de la France». Le problème évidemment, est l'identification de cette totalité, de cet effet total auquel on se réfere. Halliday remarque (p. 339):

Il n'est pas difficile de trouver des éléments proéminents organisés dans un poème ou un texte en prose, des régularités dans les sons, les mots ou les structures qui ressortent en quelque sorte, ou qu'une lecture attentive peut révéler; et cela peut souvent conduire de cette manière à une compréhension nouvelle, quand on voit qu'un tel relief contribue à la signification totale voulue par l'auteur.

C'est sur ces derniers mots que je voudrais attirer l'attention. Halliday les place en dernier, mais logiquement ils devraient venir en première position. Quelle que soit l'attention qu'on apporte à une lecture, on ne voit la signification que de certains types d'emphase - de mise en relief ou de valorisation (foregrounding or underscoring, pour utiliser deux termes en usage qui désignent la même chose) - si l'on a déjà acquis une appréciation de la stratégie d'ensemble de l'auteur. Le problème est de savoir comment distinguer entre les traits linguistiques significatifs et ceux qui sont esthétiquement sans importance, ce qu'on peut appeler le probleme de McGonagall. (M.A.K. Halliday rapporte comment le professeur Sinclair analysait les traits syntactiques du poète écossais William McGonagall -- un poète notoire-

4. Cf. Chatman (1971), p. 368. 
ment mauvais - et démontrait que son langage était tout aussi structuré que celui de n'importe qui d'autre.) Richard Ohmann, dans Literature as Sentences, me semble aborder ce problème quand il tente de démontrer le génie de James Joyce dans une phrase complexe d'A raby et celui de Conrad à partir de la dernière phrase de The Secret Sharer. On peut trouver des enchâssements tout aussi complexes dans des textes de prose dénués de prétention littéraire, et il est douteux qu'une telle approche soit satisfaisante, à moins que la pertinence stylistique des traits relevés ait été fermement établie.

René Wellek fait observer à cet égard que l'analyse littéraire commence là où s'arrête l'analyse linguistique (p. 417) ${ }^{5}$, mais cette formulation a pour effet de suggérer, involontairement peut-être, un ordre des opérations. La formulation de Riffaterre me semble plus heureuse:

Une analyse purement linguistique d'un ouvrage littéraire ne donnera que des éléments linguistiques . . cette application demande un tri préliminaire. Il est nécessaire de rassembler d'abord tous les éléments qui représentent des traits stylistiques, et de les soumettre seuls ensuite à l'analyse linguistique, à l'exclusion de tous les autres (stylistiquement non pertinents). Alors, et alors seulement, on évitera la confusion entre style et langage (p. 412).

Edward Stankiewicz résume adroitement la question comme suit (Sebeok, p. 81):

... l'étudiant en poésie n'est pas en position de décrire et d'expliquer la nature du langage poétique à moins de prendre en considération les règles du langage qui déterminent son organisation, tout comme le linguiste ne peut comprendre correctement les formes de l'expression poétique à moins de considérer les forces de la tradition et de la culture... La compréhension et l'explication... sont toujours une question de perspicacité et d'intuition...

Darbyshire suggère (p. 102) que le lecteur peut identifier une structure qui est un signal contenant un thème énonçant une politique ou un principe de sélection. Le flou de sa terminologie obscurcit légèrement une approche fondamentalement saine, à savoir la recherche d'une stratégie générale par laquelle l'auteur

5. Op. cit: Style in language, éd. Sebeok. 
s'efforce de rendre l'effet choisi (il faut bien sûr supposer que le lecteur a tout d'abord senti cet effet). On considère alors le déploiement de ce plan stratégique dans ses divers dispositifs linguistiques tactiques.

Revenons un instant à la question de la totalité. Il est clair que le terme doit s'entendre de façon relativiste. Comme les termes symbole et signe et par ailleurs comme les termes stratégie et tactique, on ne peut le quantifier autrement qu'arbitrairement. Ce n'est pas parce qu'il se préoccupe de littérature au niveau de la phrase que je trouve des raisons de critiquer Ohmann: une phrase ne peut pas être une totalité esthétique. Pourtant, pragmatiquement parlant, on peut comprendre pourquoi les critiques littéraires modernes sont fascinés par l'idée d'une linguistique qui transcenderait la phrase. D'un autre côté, on comprend aussi pourquoi la préférence française pour l'expression «textes littéraires», face au terme «littérature», a eu tant d'influence, suggérant comme elle le fait (1) que chaque oeuvre constitue son propre univers, (2) que chaque oeuvre est le produit d'une politique particulière, et (3) qu'il faudrait aborder chaque oeuvre avec une méthode critique spéciale appropriée à son caractère spécifique.

John Searle, dans Actes de langage, M.A.K. Halliday, dans son article sur William Golding lors du symposium de Chatman (1971), et A.E. Darbyshire, dans A Grammar of Style, me semblent tous indiquer l'approche correcte, celle d'un examen de plusieurs niveaux, basé sur une évaluation des traits linguistiques à la lumière d'une appréciation intuitive de l'intention de l'auteur (ou, pour éviter un mot peu populaire, de sa stratégie), évaluation qui, cela va sans dire, fait appel au talent, à la connaissance et à la croyance.

Searle distingue trois niveaux ou trois types d'actes de langage, le niveau de l'énonciation (morphophonémique), le niveau propositionnel (référence/prédication), et le niveau illocutionnaire qui est le niveau d'acte de langage proprement dit (il se ramène à demander, ordonner, souhaiter, déclarer, etc.). Halliday fait une distinction qui est plus simple à manier entre le niveau idéationnel et le niveau interpersonnel - correspondant en gros à la distinction traditionnelle entre les aspects respectivement représentationnel et expressif de la communication. Mais il 
est très important, quand on considère un texte littéraire, de pouvoir classifier le comportement de l'auteur de façon plus précise qu'en parlant, par exemple, de poésie pure ou de poésie didactique, etc., et cela semble argumenter en faveur du développement indispensable d'une terminologie pour le troisième niveau, celui des actes de langage. Une grande partie de la poésie, (on est tenté en particulier de se référer à certaines des productions les plus ésotériques de Dylan Thomas) ${ }^{6}$, est proche de la nature du «happening». On se rappelle le commentaire de Chesterton sur le poème de Browning Nationality in Drinks, où il disait que Browning façonnait là de toute évidence un curieux colifichet, (même s'il n'est pas douteux que certains membres des sociétés de Browning y voyaient une analyse merveilleusement subtile de la question du Schleswig-Holstein). On pense aussi aux remarques de conclusion de W.K. Wimsatt dans Style in Language:

But surely a poem is a verbal engine, a verbal expression, which has no end except to be known. That is its end.

Ou on pourrait se référer à la remarque de Dylan Thomas: "Les images sont ce qu'elles disent, non ce qu'elles représentent." C'est à la lumière de tels commentaires sur l'autonomie du poème que l'on peut risquer le terme de performatif de façon ad boc pour certain type de poésies, celles qui constituent simplement leur propre raison d'être. Sans avoir recours au jargon, cependant, on a besoin de se poser la question de savoir précisément ce que veut l'auteur. «Écrire un poème» est une expression qui recouvre toute une variété d'activités: exhortation, persuasion, mise en garde, souhait, exécution d'un rite, etc.

Prenant les textes plutôt que la phrase comme unité opérationnelle, et partant du postulat que nous avons toujours le choix de dire, à propos du même trait, soit "cela s'écarte du modèle» ou «cela constitue un modèle», je suggérerais la procédure suivante:

(1) Lecture attentive qui dégage l'effet général.

(2) Considération de la stratégie de l'auteur et de son intention d'ensemble.

(3) Examen des traits linguistiques pour découvrir ceux qui sont spécialement mis en relief et contribuent directement à cet effet. (Si l'on compte les

6. Par exemple Artamuise by Owllight. 
fréquences, il est évident qu'il faut prendre en considération le thème dans son rapport avec les items lexicaux.)

(4) Modification - ou éventuellement confirmation - de l'opinion initiale que l'on s'était faite de la stratégie de l'auteur d'après cet examen plus approfondi au niveau tactique.

Cette dernière opération a de toute évidence un caractère herméneutique.

Dans ce cadre de travail, et dans la section (3), les niveaux à considérer en termes de norme et de déviation, (on entendra le terme norme dans le sens déjà expliqué, c'est-à-dire en relation avec l'intention artistique de l'ensemble), sont les suivants: je ne demanderai pas un nombre déterminé de niveaux - l'interpénétration tend naturellement à militer contre toute classification systématique - , mais il faut considérer tant les traits caractéristiques de chaque niveau que ceux que soulève l'interaction entre les niveaux. Spencer et Gregory donnent un exemple utile tiré d'Othello:

Othello's cry of «Devil!» (Act 4, sc. 1, ligne 236) is a sentence, which consists of one clause, which consists of one group, itself consisting of one word which in turn consists of one morpheme ( $p$. 78).

L'exclamation d'Otbello "Diable!» (Acte 4, scène 1, ligne 236) est une phrase qui consiste en une proposition, qui consiste en un groupe, consistant lui-même en un mot qui à son tour consiste en un morphème ( $\mathrm{p}$. 78).

L'exemple sert à illustrer l'arbitraire de la distinction en niveaux dans tout cas particulier, et il suggère un trait important de l'expression poétique - particulièrement en poésie —, celui de la concentration, qui soulève continuellement des difficultés sémantiques.

Les niveaux tactiques que j'ai mentionnés peuvent s'illustrer brièvement.

En raison de la nature concentrée de la poésie, il est plus facile de trouver des exemples qui illustrent seulement un niveau d'anomalie en prose.

(1) En commençant par le niveau morphophonémique, on remarque que, dans le roman (et dans le théâtre), de telles 
anomalies sont souvent associées à la description des personnages, aux distinctions dialectales ou de classe. On peut donner en exemple le dialecte écossais du «testy medical man» du chapitre 11 de Bleak House (p. 139):

"Air you in the maydickle prayfession yourself, sir?" ou les paroles illettrées d'Anastasia Piper lors de l'enquête (p. 147-8):

orginatinin, feariocious, Methoozellers, repeatually, ou celles de Jo, le balayeur:

Never beerd of sich a think (p. 148).

On peut noter que Dickens cherche fréquemment à donner aux particularités phonétiques soit un caractère d'euphonie (feariocious, repeatually, wexed and worrited (p. 147) ont un timbre plaisant), soit une sorte de plausibilité morphémique grotesque (mots ou assemblages quasi lexicaux) comme dans maydickle, prayfession, depairture (p. 139), wepping (p. 148). Les «mots» de ce genre donnent libre cours à l'improvisation étymologique, de même que les bizarreries morphémiques comme Jabberwocky ou Finnegans $W$ ake, bien que là, évidemment, les considérations sémantiques soient plus importantes. On peut noter qu'en termes strictement phonétiques, bien des déviations de Dickens ne sont pas justifiables. Le cas classique est probablement celui du mot victuailles (victuals). La forme vulgaire vittles est attribuée presque sans distinction chez Dickens aux personnages des classes inférieures; mais étant donné que c'était là plus ou moins la prononciation standard de ce mot, la forme anomale constitue une sorte d'insulte gratuite. Dans le texte en question, pour prendre un ou deux exemples simples, Methoozellers est une distorsion non nécessaire: il est très peu vraisemblable que $\mathrm{Mme}$ Piper, des quartiers populaires de l'est de Londres (« cockney»), prononce un « $\mathrm{r} »$ final marqué dans le mot, et, cela mis à part, cette orthographe anormale serait prononcée selon toute probabilité comme l'orthographe normale. La convention qui est à la base de telles formes, celle d'une indication de vulgarité purement graphologique, mériterait d'être étudiée pour elle-même.

En poésie, les déviations de nature phonétique et morphémique sont assez répandues: on allègue habituellement un prétexte historique ou culturel pour les déviations qui sont justifiées par des considérations d'euphonie: on peut mentionner les archaïsmes 
comme ceux de Spencer. Un coup d'oeil au glossaire de la Standard Oxford Edition illustre admirablement la façon dont les déviations créent une nouvelle norme.

(2) Au niveau référence/prédicat, ce sont les traits lexicaux et syntaxiques qui nous intéressent, et leurs aspects dénotationnels et connotationnels. Les possibilités de déviation sont considérables, mais elles sont, comme le note Stephen Ullman, bien plus larges dans la sphère lexicale que dans la sphère syntaxique. En poésie, on s'attend à certains types d'écarts par rapport au langage ordinaire: un poète comme Dylan Thomas cherche à trouver des déviations créatrices de normes dans une zone langage-usage où les anomalies sont facilement assimilées par les lecteurs à la «compétence littéraire» développée. Sa technique, comme le suggère la description que fait $\mathrm{P}$.J. Wexler de la tâche du critique, est de «faire la chasse aux règles et à travers elles aux exceptions, et à travers celles-ci aux règles pour rompre les règles ${ }^{7}$.

Considérant la complexité des problèmes qui se posent face aux tentatives de mise en rapport des considérations syntaxiques et sémantiques, il est instructif de réfléchir à la façon dont on utilise chacun de ces aspects tour à tour pour essayer de comprendre les poèmes les plus obscurs de Thomas, un petit progrès d'un côté jetant de la lumière sur l'autre ${ }^{8}$. La combinaison nom abstrait + verbe, si fréquente en poésie qu'elle en est le trait normal, est peut-être l'exemple classique d'une déviation au niveau référence-prédicat:

Faith in their hands shall snap in two,

And the unicorn evils run them through.

Wallace Chafe, à partir d'exemples comme la chaise a ri, argumente que le verbe est sémantiquement central et le nom, périphérique - en contradiction avec l'axiome de Chomsky, selon lequel c'est le nom qui choisit le verbe: si l'on est forcé de donner un sens à de telles structures de surface, on interprète le nom non animé comme s'il était animé. On n'interprète pas le verbe $a$ ri de façon différente «que s'il était une différente sorte d'activité réalisée par des objets inanimés» (p. 141).

7. Essays in Criticism, 1966.

8. Cf., surtout, W. Nelson Francis: Syntax and Lit. Intern., Chatman, 1967, p. 209 ss. 
(3) Le dernier niveau que je veux introduire, le niveau illocutionnaire, se comprend le mieux comme le point de vue à partir duquel le lecteur cherche à classifier le type particulier d'activité dans lequel l'auteur s'est engagé; et peut-être aussi le point de vue à partir duquel l'auteur, sa production conative totale mise de côté (son oeuvre prise comme un tout), agit pour ainsi dire in propria persona. Les exemples les plus évidents d'interpénétration entre ce niveau et le niveau précédent (celui de la référence/prédication), «l'univers interne» de l'oeuvre, se produisent lorsque l'auteur se prend à "passer de l'autre côté du rideau» au cours du roman et à s'adresser «directement» au lecteur. L'auteur peut ainsi intervenir dans l'univers du discours qu'il a lui-même créé, en considérant toujours, bien sûr, l'effet total. Le problème que pose ce genre d'interférence (l'Erfremdungseffekt de Brecht en est une variante) est semblable à celui que soulève le caractère évidemment métalinguistique des phrases non grammaticales utilisées à des fins d'illustration. Est-ce que les références de Thackeray à ses marionnettes dans Vanity Fair sont intérieures ou extérieures à l'univers du discours? Si elles y sont intérieures, que dire des essais dans Tom Jones? On pourrait prendre en exemple la mort célèbre de Jo au chapitre 47 de Bleak House:

Dead, your Majesty. Dead, my lords and gentlemen. Dead Right Reverends and Wrong Reverends of every order. Dead, men and women, born with Heavenly compassion in your hearts. And dying thus around us every day.

C'est l'effet paradoxal de la de te fabula. Cette histoire d'un enfant fictif des bas quartiers est en même temps celle de la réalité. Il y a un changement d'univers du mot dead au mot dying.

Ce serait en général une erreur que d'associer la persona qui «représente» l'auteur dans un texte littéraire à l'écrivain luimême: mais en tout cas, l'interférence d'un niveau à un autre constitue une anomalie. Chez Fielding, chez Thackeray ou chez Dickens, cette anomalie est de fait si fréquente qu'elle en devient la norme. Somerset Maugham désapprouve entièrement des interférences de ce genre:

You don't want to hear what the author has to say on some moral or social point; you want him to get on with his story ${ }^{9}$.

9. Cf. Ten Novels, Heineman, 1954, p. 36-7. 
Une opposition simple intérieur/extérieur entre l'auteur qui fait intrusion et ses personnages demande à être bien qualifiée, comme le démontre longuement Käte Hamburger dans son livre important Die Logik der Dichtung ${ }^{10}$.

Je terminerai par les commentaires généraux suivants:

(I) - Il est nécessaire de considérer à quel point les déviations littéraires par rapport au langage ordinaire sont "négatives". Ainsi, l'inversion du sujet et du verbe en poésie peut être surtout une question d'euphonie et de considérations métriques.

Lorsque les effets de l'auteur sont délibérément en contrepoint par rapport au langage ordinaire, on peut parler de déviation "positive». Dans la poésie moderne, surtout celle qui a été influencée par Browning, cette dernière attitude est très répandue.

(II) - Parmi les écrivains en prose, Dickens est un exemple frappant. J'ai suggéré ailleurs ${ }^{11}$ que la préoccupation de Dickens pour le grotesque se réalise en grande partie en combinant le réaliste et le troublant ou l'étrange:

«Chaymaid!» cried the waiter. "Gelen box num seven wish see room!»

«Stay!» said Clennam, rousing himself. "I was not thinking of what I said. I answered mechanically. I am not going to sleep here. I am going home.»

«Deed sir? Chaymaid! Gelen box num seven, not go sleep here, gome $^{12}$.

(III) - L'une des techniques de Dickens pour réaliser cet effet sur le plan linguistique est l'anaphore ${ }^{13}$. Après avoir établi une association dans l'esprit du lecteur, par exemple entre $\mathbf{M}$. Tulkinghorn et la décoration de son plafond qui représente l'Allégorie (Allegory), Dickens peut tout au long du livre faire allusion, d'une façon élaborée et énigmatique, à $\mathbf{M}$. Chadband et à l'Allégorie:

10. Ernest Klett Verlag, Stuttgart, 1957 (2nd Ed. 1968).

11. Cf. The Grotesque in English Literature: O.U.P., 1965, p. 223.

12. Cf. Little Dorrit, chap. 3.

13. Ce n'est en aucun cas le seul expédient de Dickens: il est, par exemple, probablement, le plus grand interprète anglais du style indirect libre. 
«Goodnight!" says Mr. Tulkinghorn; and goes home to Allegory and meditation ${ }^{14}$."

C'est un exemple simple de la technique, extrêmement développée chez Dickens, du leitmotiv, et on peut voir ici qu'elle a des implications générales.

Dickens trouve une satisfaction tant dans le fait que, considérées in vacuo, de telles références produisent un effet étrange et étonnant, que dans le fait que, dans le contexte général de l'oeuvre, elles sont justifiables sémantiquement en raison de leur ancrage anaphorique. En d'autres termes, la clé du problème de la grammaticalité en littérature est souvent la sensibilité au contexte (context sensitivity), au sens étroit que des déviations apparentes (les corbeilles à papier en flammes qui peuvent ronfler furieusement dans des contes du genre de ceux d'Andersen, les gens qui habitent au nord du pôle nord dans des fantaisies de Carroll, M. Tulkinghorn «going home to Allegory and meditation») peuvent se justifier dans le contexte des «mondes possibles» dans lesquels ils se produisent; et aussi, dans un sens plus large, que, considérés en termes de ce «monde possible», de cet univers du discours, plutôt que du point de vue du langage ordinaire dont elles constituent des déviations, les formes apparemment non grammaticales n'ont structurellement rien d'exceptionnel.

On peut pousser loin les commentaires pragmatiques sur les énoncés énigmatiques, sensibles au contexte (context sensitive), basés anaphoriquement sur des métaphores et faisant fréquemment appel à des suppressions au niveau de la structure de surface, et il n'est pas question d'entreprendre ici l'examen en détail de tels matériaux. Il est clair néanmoins, qu'il y a une grande différence entre une telle approche concrète et une approche fondée sur une macrolinguistique et les règles de sélection restrictive extrêmement élaborées qui seraient nécessaires pour la classification des textes littéraires. On pourrait peut-être, en faveur de l'approche recommandée ici, arguer qu'elle «a l'avantage, comparée aux philosophies des faiseurs de systèmes, d'aborder ses problè-

14. Cf. Bleak House, chap. 11. 
mes un par un, au lieu d'avoir à inventer d'un seul coup une théorie en bloc de l'univers entier ${ }^{15}$ ».

Département d'anglais

Université de Trondheim

Norvège

\section{BIBLIOGRAPHIE}

AUSTIN, J.L.: How To Do Things With Words: Clarendon Press, 1962.

CHAFE, Wallace L.: Meaning and the structure of language: Univ. of Chicago Press, 1970.

CHATMAN, Seymour: Literary Style: a Symposium (ed.): O.U.P., New York, 1971.

CHATMAN, Seymour and LEVIN, S.R.: Essays on the Language of Literature (ed.): Houghton Mifflin, Boston, 1967.

CHOMSKY, Noam: Some Observations on the Problems of Semantic Analysis in Natural Languages: (Sign, Language, Culture, Mouton \& Co., 1970).

CHOMSKY, Noam: Selected Readings (ed.): J.P.B. Allen \& P.V. Buren: O.U.P., 1971, spec. p. 100-127.

*DARBYSHIRE, A.E.: A Grammar of Style: Andre Deutsch, 1971.

ENKVIST, Nils Erik, SPENCER, John, GREGORY, Nichael J.: Linguistics and Style: O.U.P., 1964.

*HALLIDAY, M.A.K.: Linguistic Function and Literary Style: Chatman, 1971 (Symposium).

HILL, A.A.: Grammaticality: Word. Vol. 17, 1961.

JACOBS, R.A. and ROSEBAUM, P.S.: Transformations, Style, and Meaning: Xerox Waltman, 1971.

KÖRNER, Stephan: Fundamental Questions in Pbilosopby: Penguin Books, 1969 , spec. p. 43 ss.

LANGACKER, Ronald W.: Review of Chafe 1970, in Language, Vol. 48, No. 1. March 1972.

LEECH, G.N.: A Linguistic Guide to English Poetry: Longmans, 1969.

LEVIN, S.R.: Poetry and Grammaticalness: (Chatman and Levin, 1967, [Essays]).

LEVY, J.: Generative Poetics: (Sign, Language, Culture, Mouton \& Co., 1970).

LYONS, John: Introduction to Theoretical Linguistics: Camb. U.P., 1968, spec. p. 147 ss.

LYONS, John: Chomsky: Fontana/Colins, 1970.

OHMANN, Richard: Literature as Sentences: College English, Vol. 27, No. 4. January 1966.

15. Bertrand Russell: History of Western Philosopby (1946) p. 862 
POSTAL, P.M.: Underlying and Superficial Linguistic Structure: Language. Penguin, Modern Psychology Readings, 1968.

QUIRK, Randolph, SVARTVIK, Jan: Investigating Linguistic Acceptability. Mouton \& Co., 1966.

*REVZIN, I.O.: Generative Grammars, etc. (Sign, Language, Culture: Mouton, 1970).

RIFFATERRE, Michael: Criteria for Style Analysis: (Chatman and Levin, 1967 [Essays]).

*SEARLE, John R.: Speech Acts: An Essay in the Philosopby of Language: Camb. U.P., 1969, spec. Chapter 2.

SEBEOK, Thomas A.: Style in Language (ed.): M.I.T. Press, 1960.

SEUREN, P.A.M.: Operators and Nucleus: Camb. U.P., 1969, spec. p. 13 ss.

STEINER, George: Extraterritorial: Faber, 1972.

WAISMANN, F.: The Principles of Linguistic Philosophy, ed. R. Harté: Macmillan \& Co.: 1965, spec. p. 34-40.

WELLS, Rulon: Meaning and Use (Psycholinguistics, ed. Saporta): Holt, Rinehart and Winston, N.Y., 1961.

WITTGENSTEIN, L.: Philosophical Investigations, transl. Anscombe: Basil Blackwell, Oxford, 1968: "grammar», spec. 497 ss.

*ZIFF, Paul: Semantic Analysis: Cornell U.P., N.Y., 1960.

ZIFF, Paul: «About Ungrammaticalness», (Mind, Vol. 73, 1964).

*Sources principales. 